\title{
Replication and Transparency of Qualitative Research from a Constructivist Perspective
}

\author{
Poorna Talkad Sukumar \\ University of Notre Dame \\ Notre Dame, IN, USA \\ ptalkads@nd.edu
}

\author{
Ronald Metoyer \\ University of Notre Dame \\ Notre Dame, IN, USA \\ rmetoyer@nd.edu
}

\begin{abstract}
The positivist or conventional notion of replication does not lend itself to qualitative research. However, defining replication in line with the nature of qualitative inquiry and its interpretive strengths can lead to many opportunities including creating multiple discourses of the same phenomenon and comparing the effectiveness of different methods. We define what a replication of a qualitative study can potentially entail and espouse that such replications should, by all means, be attempted. Towards these goals, we also advocate open practices in qualitative research to make more apparent the iterative and emergent processes typical of qualitative studies that are often indiscernible from their published reports.
\end{abstract}

\section{KEYWORDS}

Replication; reproducibility; qualitative research; transparency; open practices; quantitative research

\section{INTRODUCTION}

Quantitative and qualitative approaches, despite being fundamentally different, are often compared and the merits of each as forms of research are constrasted. Generally speaking, quantitative and qualitative approaches exemplify the positivist and constructivist paradigms, respectively. The positivist perspective assumes that reality or knowledge exists as the objective truth independent of those who study it while the constructivist perspective views knowledge as subjective, constructed through interaction and inseparable from those who study it [6](p.37). Hence findings of quantitative studies can be replicated and generalized to larger populations whereas qualitative findings only hold for and describe the individual cases. 
Qualitative research encompasses methods to study social phenomena from a broader perspective to generate "rich and deep" data [7] (Ch. 1). It is inherently interpretive, emphasizes context, is emergent and iterative, views the phenomenon under study "holistically", and involves dialectical and reflective reasoning [7](p. 3). Given these distinguishing characteristics, many of the terms used in reference to quantitative methods have been replaced by alternatives that are more suited to the nature of qualitative research. For example, Lincoln and Guba [6] present notions of credibility, dependability, confirmability, and transferability for qualitative inquiry which are analogous to the validity, reliability, objectivity, and generalizability constructs, respectively, applied to quantitative approaches [20].

However, replication is still applied in the positivist sense to qualitative research and considered ill-suited for it. Replication needs to be redefined for qualitative inquiry based on its inherent characteristics and interpretive strengths. Given that the constructivist approach views knowledge not as the objective truth but instead as constructed realities, the goal of replication should likewise be altered to study how these constructed realities can differ and generate "a richer and deepened" understanding of the phenomena and researcher biases instead.

We provide a definition below of what a replication of a qualitative study can potentially mean and entail. Our idea of a replication study essentially encompasses repeating some aspect of an earlier study's design and a subsequent interpretive comparison. Replication studies will be novel, standalone studies as well given the inherent influence the researchers conducting a study have on their findings.

A replication is a (novel) qualitative study conducted by independent researchers replicating one or more aspects (such as study design, research questions, context, methods, and participants) of an earlier qualitative study and embedding within its findings an

interpretive comparison with a view to corroborate, elaborate, contrast, or clarify the elements corresponding to the replicated aspects with those of the earlier study.

Replications have varying definitions in quantitative research. They range from strict to conceptual replications and can also include replications of only conditions of prior studies $[5,18]$. We similarly do not promote a rigid approach and our definition is fairly open-ended so that it can include the replication of any aspect of a study's design as well as be applied within the various genres of qualitative inquiry. However, we require that replications be conducted by independent researchers to make possible independent scrutiny in the interpreted comparisons.

Our idea of replication can be considered as being similar to the concepts of triangulation [12] and crystallization $[3,15]$ in that they all represent ways to strengthen a study's design and findings, promote a deepened understanding of a topic, and urge the researchers to reflexively consider themselves and their roles in the inquiry. Triangulation refers to the process of combining different data sources, methods, researchers, or perspectives in the study of the same phenomena [12](p. 187). Crystallization, proposed as an alternative to triangulation, is defined in reference to the qualitative 
continuum. The qualitative continuum ranges from art on one end and science on the other and situates the diverse genres of qualitative inquiry in between along with their respective representational forms including poetry, personal essays, and academic reports [3](Ch. 1). Crystallization connects multiple points in the continuum by combining the analysis methods and representational forms of multiple genres to describe the same phenomenon.

The main goal of triangulation and crystallization, however, is to combine and consolidate multiple perspectives into a coherent and rich account which also has the advantage of increasing the credibility or confidence in the accuracy of the findings [7, 12]. Replications, on the other hand, can be seen as focusing on highlighting and reflecting on the similarities and differences in the perspectives rather than on combining them, thereby also allowing discordant views to exist for a given topic.

We further illustrate the merits of replications by listing example scenarios where they can be useful and the potential advantages they bring to qualitative research in the following section. We then make a case for employing open practices in qualitative research. Making the decisions, rationales, and procedures underlying a qualitative inquiry more transparent can not only enable reviewers to assess the rigor of studies and the aptness of comparisons in replications but can also provide guidance to researchers for learning from and replicating such work.

\section{WHY CONDUCT REPLICATIONS OF QUALITATIVE RESEARCH}

Qualitative inquiry is not one defined approach but encompasses a myriad of approaches. These approaches are organized into varying genres based on factors including their theoretical and empirical underpinnings and representational forms $[3,7,16]$. We can see replications being applied in many of these genres and present a few example scenarios below. In addition, we list some general advantages and opportunities that replications can bring to the realm of qualitative research.

\section{Example Replication Scenarios in Different Qualitative Genres}

Phenomenological Approaches. These approaches study "lived experiences" of individuals [7], that is, their experiences, interpretations, and perceptions in reference to a particular phenomenon, such as an emotion, culture, or relationship [12]. Phenomenological approaches are built on the premise that there is an "essence" to such experiences and seek to capture that essence.

To analyze experiences, phenomenologists are known to adopt methods that enable them to experience the same phenomenon because, as described by Patton [12] (p. 70), "the only way for us to really know what another person experiences is to experience it for ourselves." Replications can serve as those methods which can both help in capturing the "essence" of experiences as well as enable us to experience the same phenomenon as the individual(s) in question. An example, albeit in art form, is the recent film Loving Vincent [21]. The film not only relays events in the life of the artist Vincent van Gogh and those surrounding his death by analyzing his letters [19] but it also does so 
entirely through paintings created by replicating his techniques and capturing the essence of how he perceived the world to enable the audience to experience it as he did.

Sociolinguistic Approaches. This genre consists of approaches that study people's communicative behaviors [7] (p. 20) in social-interaction settings including those involving in-person communication and technological platforms [13]. Peräkylä [13] describes a continuum of the types of empirical materials collected in qualitative research as lying between interviews or responses invoked in reference to the researchers' queries on one end and naturally occurring materials, such as talk occurring on a social-media platform, on the other end. The materials collected in this genre typically fall under the latter category.

We perceive the approaches in this genre to be good candidates not only for replications but also for reproducibility. We define reproducibility in the constructivist sense as the alternative analyses of the gathered data and also including interpretive comparisons of the findings. The comparisons can pertain mostly to the data, which is largely free from researcher instigation and represents the direct object of inquiry (as opposed to indirect or elicited from or recalled by participants), without having to factor in researcher influences in the data-collection process.

Critical Genres. There are a number of critical and emerging genres in qualitative research, such as feminist theories and participatory action research [7] (Ch. 2). They critique the power relations prevalent in traditional research where the subjects of inquiry, especially those belonging to oppressed groups, are given passive roles. Hence these genres entail approaches with empowering goals and actively involve and collaborate with the subjects of their research. Furthermore, the approaches in this genre are based on the premise that all qualitative findings embody the researchers' biases and judgments and advocate that these biases should be leveraged by researchers in their knowledge production [11](p. 28).

Given the central role played by researcher biases in these approaches, the purpose of replications in such genres can also include articulating and comparing the biases, prejudgments, and predilections of the different researchers, how they affect and shape their respective narratives, and what their stances mean for the research in question. This would require that the researchers' biases and thought processes throughout the study are made more transparent and we elaborate on this transparency aspect in the next section.

\section{General Opportunities}

Alternative to Transferability. Transferability refers to the ways in which a study's findings can be applied to other similar contexts or participants. This responsibility usually lies with the researchers intending to make the transfer rather than the original researchers and similarity (between contexts or participants) is generally determined by scrutinizing the study's theoretical framework, research 
questions, design, context, and participants [7](p. 252). However, the notion of transferability is criticized because qualitative findings are inherently bound to the individual contexts studied.

Rather than directly applying the findings to a different setting, a more beneficial approach would be to replicate the study design for the new target context or participant group. Replications and the resulting comparisons can be justified using the same similarity argument used in the case of transferring the findings.

Comparing Research Methods. The findings of studies and the knowledge produced are dependent on the methods employed, both in the collection and analysis of the data [9]. Methods can be considered as "bounded opportunities" [9] with every method having both advantages and limitations. The use of multiple methods is generally advocated to study the same phenomenon since they can be complementary and offset each others' limitations leading to more confidence in and credibility of the findings. For example, in the software development domain, surveys are routinely employed to gather supplementary numerical data corresponding to the qualitative findings of contextual inquiries and exploratory lab studies [10].

Replications can not only lead to insightful comparisons between the findings of studies, but, by employing different sets of methods, can also compare and illuminate the rigor of the methods used, which methods complement one another, and which methods are potentially more useful for the study in question. Hence replications can contribute to the knowledge on the effectiveness of different methods which can be leveraged by future studies.

Comparing Changes in the Real World. One of the objections to replicating qualitative research is that the real world changes and is being continuously constructed and hence any attempt to replicate is futile [7] (Ch. 10). On the contrary, replications can actually serve as a means to study how the real world has changed (in reference to a particular phenomenon) since the original study.

While not strictly a qualitative study, a recent study in gesture research involving qualitative data and analysis [17] replicated an elicitation study conducted nearly thirty years ago for devising gestures for text editing on tablet devices. One of its main goals was to compare the gestures obtained from participants (manually coded and grouped) in the replication with those of the earlier studies considering the advancement of technology since then. The authors present their comparisons by reflecting on the participants' mental models and the potential influences contributing to them in each case.

Reinforcing Practical solutions. Developing a practical knowhow or generating design requirements is a goal of many qualitative approaches and studies. For example, participatory action research endeavors to develop sustaining solutions to the issues faced by the participants of inquiry and in collaboration with them [14](p. 1). Numerous papers published in the human-computer interaction 
$(\mathrm{HCl})$ domain encompassing qualitative studies include an "implications for design" section wherein their key qualitative findings are converted to practical design guidelines.

While standalone qualitative studies can be useful to obtain an understanding of the topic covered, their utility in serving as the sole foundations for making sustaining changes or informing the design and development of new interventions can be questioned. There may be a need to reinforce the abstracted practical knowledge by means of multiple studies and replications can prove to be useful in this regard.

Providing Learning Opportunities. Developing the competence to design, conduct, and present qualitative studies can be achieved mostly through experience. Replications can present opportunities to students and beginners starting out in the field for developing the essential skills of identifying the relevant details to capture in their studies. We discuss how open practices in qualitative research can further contribute to providing guidance in the next section.

\section{TOWARDS OPEN PRACTICES IN QUALITATIVE RESEARCH}

Published reports and papers of qualitative studies are hardly representative of the actual processes; they often describe the processes in a linear fashion chronically ordering the steps involved with each step logically leading to the next [7](Ch. 1). Qualitative studies, in reality, tend to be far from linear processes and are typically iterative, emergent, and evolving where researchers go back and forth in making decisions concerning their studies.

Additionally, qualitative findings take the form of rich, engaging narratives which seem to appear by "magic" [7]. These rich treatises reflect the expert intuitions of researchers who, through experience, have learned the ineffable skills of competently designing and conducting qualitative studies and giving thorough attention to every relevant detail.

This elusiveness in how qualitative studies are typically communicated can hinder efforts to see the true nature of the processes, assess their rigor and usefulness, and learn from and replicate them Open practices in qualitative research can overcome these limitations and we discuss below specific purposes they can serve and how they relate to replications. We then present a checklist of general guidelines for ensuring transparency gathered from existing literature that researchers can consider towards both strengthening their studies and enabling others to learn from or build upon them.

\section{Reasons for Transparency}

Understanding Theoretical Underpinnings and Researcher Biases. The genres of qualitative research are driven by different theoretical and empirical foundations and define varying roles for researchers in the inquiry. For example, while many of the traditional approaches require that the researchers take neutral stances in their studies, the critical and emerging genres view researcher biases as being key 
to acquiring new knowledge [7](Ch. 2). Therefore, making the conceptual framework and researcher subjectivities in a study more transparent can help identify these differences and gain a comprehensive understanding of these aspects. This will also ensure reasonable comparisons of these aspects in the case of replication studies.

Assessing Similarity. Researchers have to provide similarity rationales when transferring a study's findings to a different setting or when borrowing or replicating the methods, research questions, or any aspect of a study's design. In other words, they have to describe how their study is similar in some sense to that of the study whose design is being replicated or findings transferred so as to justify the replication or transfer. To ascertain similarity, it is necessary that the earlier study's context, methods, and rationales for the choice of methods are thoroughly explained and transparent so that a robust argument can be constructed grounded in these details.

Assessing Rigor. Unlike quantitative research, where the relation between replication and validity of studies is well known [4, 18], the similar relation between replication and "rigor" of qualitative studies is not clear. This is probably because the notion of rigor itself is ambiguous for qualitative studies.

There is much debate among qualitative researchers on how to assess the rigor of studies (and also if it should be assessed) as well as what constitutes rigor. Rigor is often used synonymously with trustworthiness, soundness, validity, goodness, and credibility and represents different criteria depending on the study being evaluated [7](Ch. 2). Cho and Trent [2] describe various definitions of validity based on the diverse qualitative genres. For example, for "truth seeking" studies which rest on the premise that there is a right answer or an accurate description of the phenomenon, triangulation and the participants' agreement with the findings as a reasonable account of their experience can be considered as criteria to assess validity [2]. On the other hand, for the "personal essays" genre, the researchers' reflexivity, that is, a self-scrutiny of their subjectivity in the study of the phenomenon and knowledge production, is an accepted criterion for assessing validity [2].

Therefore, the evaluation of rigor is tailored to individual studies and generally achieved by examining the full research process, from the design phase to how the findings are reported [7]. This requires that all these steps are made transparent and available for critique.

Providing Guidance. By systematically documenting and describing their decisions, rationales, and procedures followed, researchers can provide guidance to others in designing their studies. Open practices can also help others replicate the work more closely and enable more reasonable comparisons in the replications. Furthermore, by making the data or evidence collected available, such as tape recordings and transcripts of conversations, researchers can enable and guide others in reanalyzing the data. 
Assessing Ethical Considerations. Ethical considerations are of paramount importance in qualitative studies and generally go hand in hand with their trustworthiness [7] (Ch. 3). Open practices can help assess the sensitivity of the researchers to the ethical issues concerning their choice of research methods, participant group, and other aspects of their studies. These ethical considerations can also guide researchers replicating the work and highlight issues they should be aware of.

\section{General Checklist for Ensuring Transparency}

Open practices and transparency in qualitative research not only entail the sharing of the collected data and supporting materials, but also include providing in-depth descriptions and explanations for the various decisions made during a study. We are aware that researchers will generally be constrained by publication page limits and ethical considerations from explaining or disclosing certain aspects of their studies. Furthermore, communicating how their findings came to be is, in many cases, not a goal of the representational forms of qualitative research which mainly aim to present rich and engaging narratives and sometimes, also to employ creative means, such as poems and multimedia texts [3] (Ch. 1). However, researchers can make these details, where permissible, available as part of supplementary material or on open-access repositories.

We list a few general guidelines below, gathered mostly from the book by Marshall and Rossman [7] and the article by Mays and Pope [8], that researchers can consider in their efforts to make their research processes and data more transparent so that the aforementioned objectives can be achieved.

(1) Clearly explain the theoretical or conceptual framework and the methods used in every stage of the research process.

(2) Discuss in detail the subjectivities and biases of researchers (both personal and theoretical biases/assumptions)

(3) Discuss how many researchers were involved in the study and their roles in each stage of the process.

(4) Discuss if any preliminary or pilot studies were conducted and how they informed the current study.

(5) Sufficiently explain the context of study.

(6) Discuss in detail the ethical issues pertaining to the study and how they have been addressed

(7) Adequately describe the participant-selection process and the theoretical basis for selecting those participants.

(8) Keep thorough notes, or a diary recording every research design decision and the rationale for it.

(9) Describe the study procedure, that is, the collection of data or evidence from participants, in detail. 
(10) Make data or evidence collected available (fieldwork notes, interview transcripts, etc.)

(11) Organize the data and researcher notes in retrievable forms.

(12) Clearly describe the procedures used for data analysis; justify their choice theoretically and how they relate to the original research questions. Make explicit how the themes and concepts were identified from the data

(13) Mention and sufficiently explain the search for or presence of "negative" or "deviant" instances or behaviors, that is, evidence that would have contradicted or modified the analysis.

(14) Present sufficient detail to bridge the gap between the interpretation or findings presented and the collected evidence by, for example, numbering quotations and labeling sources.

\section{CONCLUSION}

We indeed began exploring this topic as a result of the increasing prominence that replication has been receiving in quantitative research. However, the purpose of this paper was not to project the positivist meanings of replication, validity, and reproducibility onto qualitative research but instead to situate them in the context of qualitative inquiry and its diverse genres.

Given our rather broad definition of replication, it is possible that such replications already exist although they may not be labeled as such. Replications can be defined in different ways as well and also depending on particular qualitative genres. However, we also suspect that researchers may have misgivings about using the terms "replication" and "reproducibility" in the qualitative context and perhaps these ideas should be represented by different terms for qualitative research.

\section{ACKNOWLEDGMENTS}

We are thankful for the valuable inputs of the participants of the break-out session at the BELIV Workshop 2018 [1] where we presented our initial ideas on the topic.

We also thank Dr. Chat Wacharamanotham for providing feedback on this paper.

\section{REFERENCES}

[1] Accessed: 12-25-2018. BELIV2018. https://beliv-workshop.github.io/schedule.html

[2] Jeasik Cho and Allen Trent. 2006. Validity in qualitative research revisited. Qualitative research 6, 3 (2006), 319-340.

[3] Laura L Ellingson. 2009. Engaging crystallization in qualitative research: An introduction. Sage.

[4] Steve Haroz and Robert Kosara. 2018. Skipping the Replication Crisis in Visualization: Threats to Study Validity and How to Address Them. In Proceedings of BELIV 2018: Evaluation and Beyond - Methodological Approaches for Visualization.

[5] Kasper Hornbæk, Søren S Sander, Javier Andrés Bargas-Avila, and Jakob Grue Simonsen. 2014. Is once enough?: on the extent and content of replications in human-computer interaction. In Proceedings of the SIGCHI Conference on Human Factors in Computing Systems. ACM, 3523-3532.

[6] Yvonna S Lincoln and Egon G Guba. 1985. Naturalistic inquiry. Vol. 75. Sage.

[7] Catherine Marshall and Gretchen B Rossman. 2014. Designing Qualitative Research. Sage publications. 
[8] Nicholas Mays and Catherine Pope. 1995. Qualitative research: rigour and qualitative research. Bmj 311, 6997 (1995), $109-112$

[9] Joseph E McGrath. 1995. Methodology matters: Doing research in the behavioral and social sciences. In Readings in Human-Computer Interaction. Elsevier, 152-169.

[10] Brad A Myers, Andrew J Ko, Thomas D LaToza, and YoungSeok Yoon. 2016. Programmers are users too: Human-centered methods for improving programming tools. Computer 49, 7 (2016), 44-52.

[11] Joyce McCarl Nielsen. 1990. Feminist Research Methods Exemplary Readings in the Social Sciences. (1990).

[12] Michael Quinn Patton. 1990. Qualitative evaluation and research methods. SAGE Publications, inc.

[13] Anssi Peräkylä. 2008. Analyzing talk and text. Collecting and interpreting qualitative materials 3 (2008), 351-374.

14] Peter Reason and Hilary Bradbury. 2001. Handbook of action research: Participative inquiry and practice. Sage.

[15] Laurel Richardson and Elizabeth St Pierre. 2008. A method of inquiry. Collecting and interpreting qualitative materials 3,4 (2008), 473.

[16] Thomas H Schram. 2006. Conceptualizing and proposing qualitative research 2 ed. New Jersey: Pearson Prentice Hall.

[17] Poorna Talkad Sukumar, Anqing Liu, and Ronald Metoyer. 2018. Replicating User-defined Gestures for Text Editing. In Proceedings of the 2018 ACM International Conference on Interactive Surfaces and Spaces. ACM, 97-106.

[18] Poorna Talkad Sukumar and Ronald Metoyer. 2018. Towards Designing Unbiased Replication Studies in Information Visualization. In Proceedings of BELIV 2018: Evaluation and Beyond - Methodological Approaches for Visualization.

[19] Vincent Van Gogh. 1958. The Complete Letters of Vincent Van Gogh (3 Volume Set). (1958).

20] Daphne C Watkins. 2012. Qualitative research: The importance of conducting research that doesn't "count". Health promotion practice 13, 2 (2012), 153-158.

[21] H. Welchman, I. Mactaggart, D. Bobbitt, S.M. (Producers) \& Kobiela, and H. (Directors) Welchman. (2017). Loving Vincent [Motion Picture]. BreakThru Productions and Trademark Films. 